\title{
Televisão, Covid-19 e Cultura: análise da cobertura de temas culturais nos telejornais durante a pandemia
}

Television, Covid-19 and Culture: analysis of the coverage of cultural themes in the TV news during the pandemic

Carla Baptista - NOVA FCSH ICNOVA - Instituto de Comunicação da NOVA carla.baptista@fcsh.unl.pt https://doi.org/10.26619/978-989-9002-14-2.1

\begin{tabular}{|c|c|c|}
\hline Recebido / Received & Aceite / Accepted & Publicado / Published \\
06.10 .2020 & 09.10 .2020 & 15.01 .2021 \\
\hline
\end{tabular}

Como citar este capítulo / How to quote this chapter:

Baptista, C. (2021). "Televisão, Covid-19 e Cultura: análise da cobertura de temas culturais nos telejornais durante a pandemia". In Lourenço, J. \& Lopes, P., Comunicação, Cultura e Jornalismo Cultural. (pp. 13-35). Lisboa: NIP-C@M \& UAL, disponível em https://repositorio.ual.pt/handle/11144/4748. DOI https://doi.org/10.26619/978-989-9002-14-2.1 


\section{Resumo}

Este artigo analisa a cobertura jornalística do tema Cultura e Espetáculos nos principais jornais televisivos dos canais generalistas (SIC, RTP, TVI), durante períodos distintos da pandemia de Covid-19. São analisados 9 dias de cobertura informativa televisiva no Jornal da Noite, Telejornal e Jornal das 8 , distribuídos pelos 3 estados definidos pelo governo face à situação do país: estado de emergência (março de 2020); estado de alerta (julho de 2020); estado de contingência (setembro de 2020). Procurámos compreender qual a narrativa emergente de crise da cultura relacionada com a pandemia produzida pela informação televisiva. A análise incide sobre o tratamento dos temas culturais, comparando a performance dos diferentes canais numa dimensão quantitativa - quanto tempo dedicado aos temas culturais e qual a share dessas notícias? -, e qualitativa - quais foram os assuntos tratados dentro da cultura e como evoluiu a seleção noticiosa ao longo dos 3 períodos? 


\section{Abstract}

This article analyzes the journalistic coverage of the theme Culture and Spectacles in the main television newspapers of the generalist channels (SIC, RTP, TVI) during different periods of the Covid-19 pandemic. We analyze 9 days of television information coverage in Jornal da Noite, Telejornal and Jornal das 8 , distributed by the 3 states defined by the government in relation to the situation of the country: state of emergency (March 2020); alert stage (July 2020); contingency state (September 2020). We seek to understand the emerging narrative of culture crisis related to the pandemic produced by television information. The analysis focuses on the treatment of cultural themes, comparing the performance of the different channels in a quantitative dimension (how much time dedicated to the cultural themes coverage and what share for these type of news?) and qualitative (what were the subjects dealt with within the culture and how the news selection evolved over the 3 periods?). 


\section{Cancelado. Adiado. Fechado. A cultura segue dentro de momentos?}

Os efeitos da pandemia de Covid-19 no sector cultural podem ser olhados de formas distintas: foi péssimo, porque cancelou as atividades culturais in situ, fechou as salas de espetáculo, destruiu empregos, deixou artistas e técnicos em grande precariedade laboral e interrompeu dinâmicas de públicos que levaram décadas a consolidar. É impossível contrapor dizendo que (também) foi "ótimo", dada a devastação que provocou na economia financeira e moral do campo artístico e criativo, mas o confinamento fez disparar os níveis de consumo de produtos culturais, particularmente filmes, música e entretenimento, assistidos via plataformas digitais. Enquanto a produção cultural sofreu cortes totais, o consumo de bens culturais ajudou a minorar os efeitos do confinamento global, contribuindo para o bem estar emocional, individual e coletivo, e para reforçar sentimentos de partilha e pertença identitária.

A transferência do consumo para os serviços online beneficiou apenas as plataformas, cujo modelo de negócio já assentava no pagamento desses serviços distribuídos on demand. Os produtores culturais, ao nível institucional (fundações, teatros, museus, cinemas, centros culturais), e ao nível individual ou de micro coletivos (artistas, bandas, companhias de dança e teatro, bibliotecas) disponibilizaram gratuitamente o seu arquivo ou ofereceram live performances nas redes sociais sem expetativa de retorno financeiro. 
As experiências de inovação tecnológica nas fases de produção, em particular produtos áudio (música) e visuais (cinema e televisão) existiram e vão provavelmente mudar, no futuro próximo, a forma como se faz música, filma, fotografa ou representa. Mas são modestas comparadas com os impactos tremendos que a pandemia provocou na relação com os públicos de cultura. Esta foi dramaticamente transformada, com o regresso às salas, aos palcos, às exposições, em geral a todas as manifestações culturais, a acontecer em formato digital only, ou híbridos, deslocando uma parte das atividades ou do público para lugares virtuais. Não sabemos (ainda) como estes modelos impactam nas formas de participação, nas sociabilidades, nas cognições, afetos e perceções, geradas pela exposição e envolvimento com produtos e processos culturais. A obsessão com a digitalização cultural, potenciada como solução principal para um futuro modelo sustentável, empobrece uma agenda de investigação e de questionamentos que devia manter no radar o estudo da diversidade de fruições, interseções e conexões entre a cultura, a política e a tecnologia.

As formas de financiamento da cultura, uma discussão sempre em aberto devido à diversidade de situações laborais e à fragilidade financeira estrutural, tornou-se uma questão de emergência. As quebras de receitas em todos os subsectores, incluindo nas vendas de livros, foram dramáticas. As restrições impostas por razões sanitárias vão prolongar essas quebras durante um longo período, devido aos limites existentes para a ocupação dos espaços. A gratuidade temporária, articulada com um medo 
social persistente e o empobrecimento generalizado devido à recessão económica provocada pelas medidas de contenção da pandemia, pode gerar um movimento de não retorno, de criadores que sucumbem e de públicos que desaparecem.

A sustentabilidade de projetos culturais com funções vitais de empoderamento cívico em meios deprimidos (situados fora dos centros urbanos ou junto de populações carenciadas) ficou ameaçada, já que os seus principais financiadores (municípios e associações elegíveis para captar fundos destinados a projetos de desenvolvimento local e regional) canalizaram os recursos para outras áreas consideradas prioritárias (saúde, segurança e apoio social de emergência).

No cenário pandémico o regresso das atividades culturais implica custos acrescidos em pessoal (de apoio, de limpeza), inviabilizando qualquer programa fora de uma articulação com os poderes públicos. Há ainda a considerar as questões intrinsecamente artísticas: como continuar a treinar competências e a criar conexão entre pessoas (atores, cantores, bailarinos, cantores, performers) mantidos à distância e privados da experiência plena dos palcos? $\mathrm{Na}$ expressão utilizada pela $\mathrm{OCDE}{ }^{1}$, o "culture shock" afetou e irá continuar a afetar negativamente uma cadeia de processos e agentes, agravando desigualdades territoriais e sociais, reduzindo a oferta e enfraquecendo dinâmicas de democratização, inclusão e fruição plena de uma vida cultural.

1 Respostas políticas da OCDE ao Coronavírus (COVID-19), Choque cultural: COVID-19 e os setores cultural e criativo, disponível em: https://www.oecd.org/coronavirus/policyresponses/culture-shock-covid-19-and-the-cultural-and-creative-sectors-08da9e0e/ 
Será que a cultura, tão bruscamente interrompida, pode seguir dentro de momentos?

\section{Cultura nos media: o papel dos jornalistas culturais}

A discussão académica sobre as dificuldades em definir o conceito de cultura é longa e não nos ocuparemos dela neste artigo. As representações jornalísticas referem-se aos usos da cultura, à cultura enquanto prática, associada a performances individuais ou de grupo. Nesta visão, a cultura é algo que as pessoas fazem, em vez de algo que as pessoas possuem. As práticas culturais são situadas e não objetivas, e dinâmicas, em vez de estáveis. A dimensão de "luta" raramente atravessa os enquadramentos noticiosos da cultura, embora seja o pano de fundo envolvendo todas as práticas sociais e discursivas construtoras e transportadoras de sentidos culturais.

Os enquadramentos jornalísticos sobre a cultura refletem uma "cultural resonance", uma "ressonância" que afeta a seleção e o tratamento dos temas, e se relaciona mais com os modos de ver e de fazer dos jornalistas, e das suas organizações, do que com as qualidades intrínsecas aos objetos e sujeitos reportados. No jargão profissional em língua inglesa, os jornalistas culturais são designados por "arts journalists", traduzindo uma opção editorial estratégica de confinar a sua área de atuação ao campo das produções artísticas. Os ainda (poucos) estudos existentes (Forde, 2003; Harries \& Wahl-Jorgensen, 2007; Hovden \& Kristensen, 2018), indicam que esta comunidade particular partilha uma certa "excentricidade" relativamente aos outros jornalistas. 
Os jornalistas que indicam a cultura como o seu "beat" principal desafiam e reconfiguram conceções existentes de jornalismo como um discurso centrado em factos relatados de forma objetiva. A lógica profissional do jornalismo cultural privilegia o lado humano das histórias, as interpretações e o estilo pessoal próximo na narrativa literária (Hellman \& Jaakkola, 2012). 0 "excepcionalismo" traduz-se ainda numa formação académica diferenciada, normalmente em artes ou humanidades, que funciona como uma fonte de legitimação acrescida para pronunciamentos opinativos sobre as matérias reportadas.

Trabalhando a partir de dados obtidos pelos questionários a jornalistas realizados pelo projeto Worlds of Journalism (Hanitzsch et all, 2019), Hovden \& Kristensen (2019) confirmam o desalinhamento dos jornalistas culturais em relação aos seus pares no que diz respeito à auto perceção dos papéis sociais desempenhados. Vigiar o poder e estabelecer a agenda política são encarados como menos relevantes do que contar histórias, entreter e promover a tolerância. Embora os jornalistas culturais sejam também um dos grupos mais vulneráveis à precariedade laboral, sobretudo os que "cobrem" as áreas ligadas ao lifestyle, aqueles que trabalham em redações dizem sentir pouca pressão de anunciantes, dos agentes culturais e do público. Os autores concluem que as editorias de cultura são espaços relativamente privilegiados dentro dos meios de comunicação social, com os jornalistas culturais a beneficiarem de níveis elevados de autonomia para proporem histórias baseadas nas suas predileções e especializações. 
O alargamento crescente de tópicos abarcados na designação genérica de jornalismo cultural, misturando artes com entretenimento e lazer (Kristensen \& Riegert, 2017), coloca-o na zona de fronteira (boundary work) que marca as práticas jornalísticas contemporâneas, oscilando entre a exigência de autonomia e a cedência a pressões comerciais. A diversificação temática, acompanhada de perda de especialização e diminuição da crítica cultural em favor da divulgação cultural, levou estudos recentes (Hellman \& Jaakkola, 2012; Sarrimo, 2017) a assinalarem a tendência para a "newsificação" do jornalismo cultural, ou seja, a sua aproximação ao cânone da profissão. A conclusão de que os alinhamentos do jornalismo cultural são cada vez mais influenciados pelos valores das notícias tradicionais é particularmente relevante neste estudo, que analisa a cobertura jornalística da cultura nos telejornais com maior audiência nos canais generalistas (SIC, RTP, TVI), durante períodos distintos da pandemia. A exposição anterior visou identificar a complexidade e multiplicidade de dimensões associadas ao lockdown cultural, passíveis de serem tratados como matérias noticiosas. Interessa-nos compreender qual a narrativa emergente de crise da cultura relacionada com a pandemia produzida pela informação televisiva.

\section{Televisão, a grande educadora}

A relação entre televisão e cultura popular é praticamente fundadora dos estudos culturais televisivos. O título do livro clássico de John Fiske (1987) - Television Culture - pretende sublinhar que a televisão não transmite cultura, mas produz cultura. A ar- 
ticulação entre televisão e Estado-nação, quebrada na história pelas dinâmicas de fragmentação introduzidas pelos novos media, não matou a televisão. Esta mostra uma resiliência e uma adaptabilidade notáveis, ao nível da oferta de conteúdos e dos modos de distribuição, mantendo-se o media com a presença mais significativa na vida das pessoas. O Digital News Report $2020^{2}$ indica que, em abril, em países onde o consumo de notícias já era maioritariamente online (a Alemanha, por exemplo), a televisão conseguiu posicionar-se, temporariamente, como a principal fonte noticiosa. A "golden age", alavancada pelas séries de qualidade que alargaram as audiências globais da televisão, deu lugar à "peak TV" e a receita parece ser sempre mais e meIhor. Hartley (2020:25) menciona que na era da televisão "pós-global" esta continua a ser líder na "produção e distribuição da subjetividade moderna".

A pandemia voltou a sentar as famílias em frente à televisão, restaurando o pleno triádico das suas funções comunicativas: informar, entreter e formar. Ong \& Negra (2020) referem que a televisão pandémica funcionou como um "timekeeper", introduzindo regularidades temporais que tinham caído em desuso. Um exemplo são as conferências de imprensa de políticos e de autoridades de saúde para divulgar as medidas em curso e os números da doença. A autora (2020:3) refere que a televisão americana durante o início da pandemia foi marcada por "uma cacofonia de figuras masculinas carismáticas", partilhando uma mistura de paternalismo, autoritarismo, compaixão e uma retórica

2 Digital News Report, disponível em: https://www.digitalnewsreport.org/ 
coerente. Em Portugal, algo de semelhante ocorreu, com as televisões a lutarem para fixar a maior share de uma super audiência, obcecada por informação relativa ao mono tema Covid-19. Entre as figuras do meio televisivo catapultadas para a dimensão do estrelato nacional estão os pivots dos telejornais do prime time.

\section{O efeito COVID-19 nos telejornais: tratamento de temas culturais}

O interesse por notícias aumentou durante as semanas de confinamento que se seguiram à declaração do estado de emergência em Portugal, em 18 de março de 2020. Segundo uma sondagem realizada pelo grupo Mediabrands ${ }^{3}$ em 23 de março, "o pico diário de audiências durante o dia (mais de 5,9 milhões de espetadores médios) dá-se entre as $20 \mathrm{~h}$ e as $21 \mathrm{~h}$, momento em que são transmitidos os jornais informativos em vários canais em simultâneo." A procura de conteúdos informativos foi um dos efeitos imediatos. Entre março de 2020 (estado de emergência), passando por julho de 2020 (estado de alerta) e setembro de 2020 (estado de contingência), o Jornal da Noite (SIC) foi o mais visto, seguido pelo Telejornal (RTP1) e pelo Jornal das 8 (TVI). Segundo dados da Cision ${ }^{4}$, que analisou as audiências dos jornais televisivos do prime time desde a semana de 3 a 9 de março (anterior ao confinamento), até à semana de 30 de junho a 6 de julho, "o bloco informativo da SIC surge destacado, a apre-

3 "Consumo de televisão sobe $28 \%$ com a pandemia de Covid-19", Diário de Notícias, 23 de março de 2017, disponível em: https://www.dn.pt/dinheiro/consumo-de-televisaosobe-28-com-a-pandemia-de-covid-19-11972940.html

4 "Audiências dos telejornais", CISION, 24 de Julho de 2020, disponível em: https://www. cision.pt/2020/07/audiencias-dos-telejornais/ 
sentar uma audiência média diária de 1325521 telespetadores, seguindo-se o da RTP1, com 883961 telespetadores; e o da TVI, com 861903 telespetadores."

Neste estudo, analisámos 9 dias de informação televisiva no prime time (das $19 \mathrm{~h}$ às $22 \mathrm{~h}$ ), nos 3 jornais televisivos com mais audiência, respetivamente o Jornal da Noite (SIC), o Telejornal (RTP1) e o Jornal das 8 (TVI). O recorte temporal contempla 3 períodos distintos, designados a partir da definição política da situação do país face à Covid-19. A amostra inclui 3 dias durante o estado de emergência (17-19 de março), 3 dias durante o estado de alerta (1-3 de julho) e 3 dias durante o estado de contingência (15-17 de setembro). Os diferentes estados traduzem a avaliação da gravidade da situação relativamente aos contágios e determinam as medidas de resposta. É expetável que o desagravamento progressivo das restrições conduza a uma agenda noticiosa mais alargada, com novos temas e protagonistas a surgirem fora do enquadramento pandémico. A análise incide sobre o tratamento informativo dos temas culturais, comparando a performance dos diferentes canais em dimensões quantitativas. Quanto tempo à cultura e qual a share dessas notícias? -, e qualitativas - quais foram os temas dentro da cultura, quais receberam mais atenção e como evoluiu a seleção noticiosa ao longo dos 3 períodos? Procuramos compreender qual foi a narrativa televisiva emergente sobre a cultura e como foi afetada pelo efeito da pandemia. Os resultados foram obtidos a partir de dados cedidos pelo e-Telenews ${ }^{5}$, um serviço da MediaMoni-

5 E-telenews, disponível em: https://www.marktest.com/wap/a/grp/p 43. aspx\#apresentação 
tor que monitoriza audiências televisivas e radiofónicas, a quem agradecemos a colaboração, que permitiu viabilizar esta pesquisa.

\subsection{Apresentação dos resultados}

O conjunto de imagens abaixo detalham os resultados obtidos para o tema de Cultura e Espetáculo. Vamos começar por analisar a share percentual das notícias sobre cultura apresentadas nos 3 jornais televisivos ao longo do tempo de monitorização definido. O quadro 1 indica que a share foi maior para o Jornal da Noite da SIC, em todos os períodos. O Telejornal da RTP1 obteve a segunda share mais alta na fase de emergência, mas tem uma oscilação descendente, enquanto o Jornal das 8 da TVI vai recuperando share e termina setembro com melhores resultados do que em março.

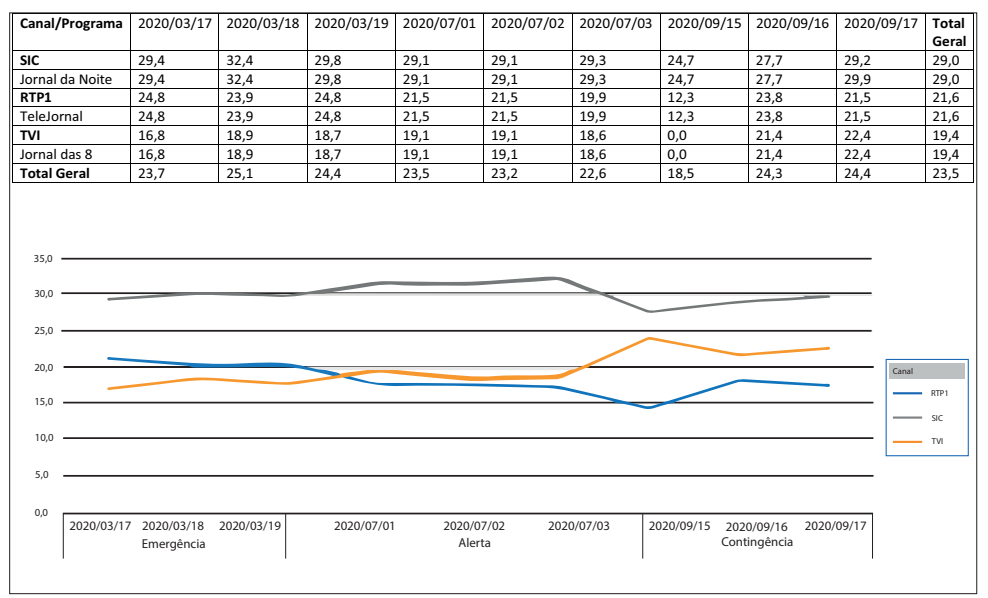

Quadro 1 Share em percentual dos noticiários televisivos do horário prime durante os períodos analisados. Fonte: e-Telenews - Elaboração própria. 
No quadro 2, a tabela e o gráfico à esquerda referem-se à quantidade de inserções relacionadas com Cultura e Espetáculos nos nove dias de análise. $\mathrm{O}$ gráfico do lado direito resume a divisão do tema Cultura e Espetáculos por assuntos, de acordo com a proporção de cobertura dada por cada canal de televisão. Ressalta que o Telejornal da RTP1 foi o que mais tratou do tema, registando quase três vezes mais inserções do que os concorrentes.

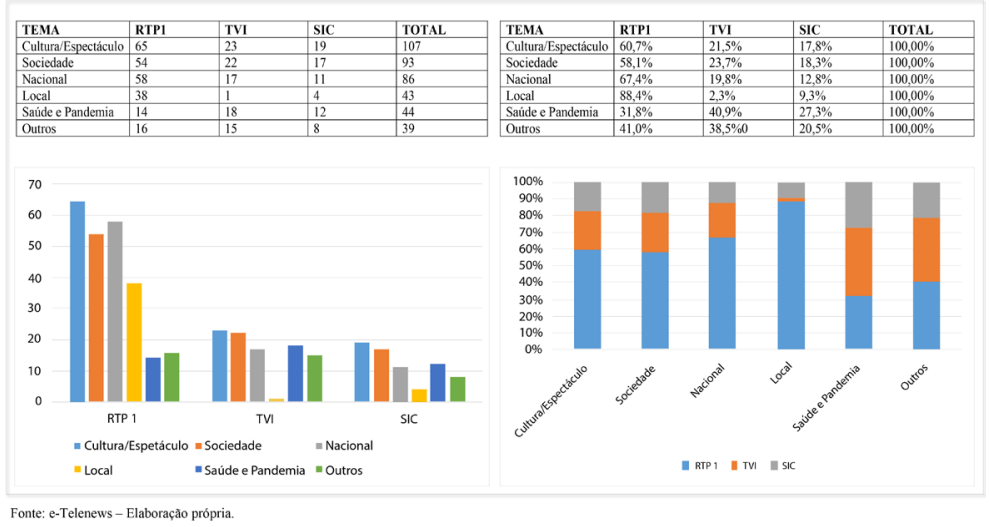

Quadro 2 Quantidade (tabela e gráfico da esquerda) e proporção (tabela e gráfico da direita) de temas associados a Cultura/Espetáculos nos noticiários Televisivos dos três canais. Fonte: e-Telenews - Elaboração própria.

Neste conjunto de tabelas e gráficos do quadro 2 é possível observar quantos minutos cada canal dedicou aos diferentes temas associados à Cultura e Espetáculo. Dos 107' dedicados a este assunto nos períodos de monitorização, 93' foram também relacionados ao tema Sociedade, e outros 86 minutos associa- 
dos ao tema Nacional. Globalmente, a associação entre Cultura e Espetáculos com notícias de âmbito Local e com notícias relacionadas com Saúde e Pandemia foi muito semelhante, embora com variações importantes na abordagem de cada canal de televisão.

Do lado direito do quadro é possível observar para cada tema associado ao de Cultura e Espetáculo o quanto estes foram proporcionalmente transmitidos pelos canais. Estes conteúdos tiveram $60,7 \%$ das inserções feitas pela RTP1, enquanto que a TVI e a SIC obtiveram, respetivamente, $21,5 \%$ e $17,8 \%$ da proporção de transmissão no período. As proporções mantiveram-se similares para outros dois temas que apareceram associados a Cultura e Espetáculo, nomeadamente a Sociedade e o Nacional. O tema Saúde e Pandemia (sempre associado à Cultura e Espetáculo) foi $40,9 \%$ das vezes transmitido pela TVI. O tema Local, sempre associado aqui ao universo de Cultura e Espetáculo, foi mais transmitido pela RTP1 $(88,4 \%)$, conforme atribuição registada na aplicação e-Telenews.

Na tabela 1 abaixo podemos observar que os diferentes canais de TV tenderam a apresentar outros conteúdos associados ao da Cultura e Espetáculos, de modo similar, ao relacionar grande parte deste universo (Cultura/Espetáculo) a temas como Sociedade $(86,9 \%)$ e a Nacional $(80,4)$. Isso indica certa similaridade sobre o modo como trataram destes temas durante os três estados da pandemia. O tema Cultura e Espetáculos e o tema Saúde e Pandemia foram mais associados no caso das notícias 
transmitidas pelo Telejornal da RTP1 (58,5\%) do que em comparação com o Jornal da Noite da SIC (21,1\%) e o Jornal das 8 da TVI (4,3\%), de acordo com a classificação feita pela e-Telenews.

\begin{tabular}{|l|r|r|r|r||}
\hline TEMA & \multicolumn{1}{|l|}{ RTP1 } & \multicolumn{1}{l|}{ TVI } & \multicolumn{1}{l|}{ SIC } & TOTAL \\
\hline Cultura/Espectáculo & $100,0 \%$ & $100,0 \%$ & $100,0 \%$ & $100,0 \%$ \\
\hline Sociedade & $83,1 \%$ & $95,7 \%$ & $89,5 \%$ & $86,9 \%$ \\
\hline Nacional & $89,2 \%$ & $73,9 \%$ & $57,9 \%$ & $80,4 \%$ \\
\hline Saúde e Pandemia & $58,5 \%$ & $4,3 \%$ & $21,1 \%$ & $40,2 \%$ \\
\hline Local & $21,5 \%$ & $78,3 \%$ & $63,2 \%$ & $41,1 \%$ \\
\hline Outros & $24,6 \%$ & $65,2 \%$ & $42,1 \%$ & $36,4 \%$ \\
\hline \hline
\end{tabular}

Tabela 1 Relação proporcional de diferentes temas comparados ao de Cultura e Espetáculo (100\%) nos noticiários televisivos dos três canais de TV analis dos Fonte: e-Telenews - Elaboração própria.

A tabela 2 (abaixo) permite visualizar como os grupos de assuntos Artistas/Personalidades, Premiações, Cinemas/Teatros/Espetáculos e Festivais/Concertos foram os mais tratados dentro do universo temático englobado por Cultura/Espetáculos.

\begin{tabular}{||l|r|r|r|r|r|r|r|r||}
\hline Assuntos & \multicolumn{1}{|l}{ RTP1 } & TVI & SIC & TOTAL & RTP1 & TVI & \multicolumn{1}{l|}{ SIC } & TOTAL \\
\hline Artista/Personalidade Específica & 9 & 2 & 4 & 15 & $42,9 \%$ & $13,3 \%$ & $11,8 \%$ & $21,4 \%$ \\
\hline Premiações & 3 & 4 & 4 & 11 & $14,3 \%$ & $26,7 \%$ & $11,8 \%$ & $15,7 \%$ \\
\hline Cinemas/Teatros/Espetáculos & & 3 & 7 & 10 & & $20,0 \%$ & $20,6 \%$ & $14,3 \%$ \\
\hline Festivais/Concertos & 7 & & 3 & 10 & $33,3 \%$ & & $8,8 \%$ & $14,3 \%$ \\
\hline TVs/Rádios & & & 9 & 9 & & & $26,5 \%$ & $12,9 \%$ \\
\hline Situação Artistas & 2 & 6 & & 8 & $9,5 \%$ & $40,0 \%$ & & $11,4 \%$ \\
\hline Museus/Exposiç̃̃es & & & 4 & 4 & & & $11,8 \%$ & $5,7 \%$ \\
\hline Caridade/Filantropia/Ação Social & & & 3 & 3 & & & $8,8 \%$ & $4,3 \%$ \\
\hline TOTAL & $\mathbf{2 1}$ & $\mathbf{1 5}$ & $\mathbf{3 4}$ & $\mathbf{7 0}$ & $100,0 \%$ & $100,0 \%$ & $100,0 \%$ & $100,0 \%$ \\
\hline \hline
\end{tabular}

Tabela $\mathbf{2}$ Inserções e proporção de notícias veiculadas por grupos de assuntos para cada canal de TV. Fonte: e-Telenews - Elaboração própria. 
A tabela e os gráficos seguintes avaliam a interseção entre os temas de cultura e o tema Covid-19 (consideramos todas as notícias onde havia referências a medidas sanitárias, estados de saúde de protagonistas da cultura, restrições na entrada de eventos culturais, obtendo assim o indicador Saúde e Pandemia, tal como indicado no Quadro 2). Na tabela 3 podemos observar que no início da pandemia houve alguma cobertura diretamente associada ao tema da saúde, mas foi durante o estado de alerta que ocorreu a maior incidência de notícias relacionando o tema Cultura/Espetáculo e Saúde/Covid-19. Esta relação deixou de ocorrer durante o período de contingência.

\begin{tabular}{||l|l|l|l|l|l|l|l|l|l|r||}
\hline \multirow{2}{*}{ Tema e Grupo de Assunto / Canal } & \multicolumn{3}{|c|}{ Emergência } & \multicolumn{3}{c|}{ Alerta } & \multicolumn{2}{c|}{ Contingência } & \multirow{2}{*}{ Total } \\
\cline { 2 - 11 } & SIC & TVI & RTP1 & SIC & TVI & RTP1 & SIC & TVI & RTP1 & \\
\hline Saúde & & & & & & & & & & 3 \\
\hline TVs/Rádios & & & & & & & & & & 1 \\
\hline Situação Artistas & & & & & & & & & & 1 \\
\hline Cinemas/Teatros/Espetáculos & & & & & & & & & & 1 \\
\hline Covid-19/Coronavírus & & & & & & & & & & 3 \\
\hline Caridade/Filantropia/Ação Social & & & & & & & & & & 1 \\
\hline Situação Artistas & & & & & & & & & & 1 \\
\hline Festivais/Concertos & & & & & & & & & & 1 \\
\hline \hline
\end{tabular}

Tabela 3 Inserções no noticiário televisivo (3 canais) sobre temas e grupos de assuntos relacionados com Cultura e Espetáculos/Saúde e Covid-19 nos 3 períodos avaliados. Fonte: e-Telenews - Elaboração própria.

Ao considerarmos somente as notícias do universo de Cultura e Espetáculo diretamente relacionadas à temática Covid-19 observamos uma ocorrência para cada um dos canais analisados. As três ocorrências de notícias foram transmitidas durante o período de alerta. Ao ampliarmos a análise, considerando o universo conjunto de temas da Cultura e Espetáculo com os temas 
Covid-19 e Saúde, observamos que a quantidade de notícias é aumentada para 6 ocorrências, sendo que 1 delas se deu durante o estado de emergência e as outras 5 durante o estado de alerta, tal como se pode observar no quadro 3 (abaixo).

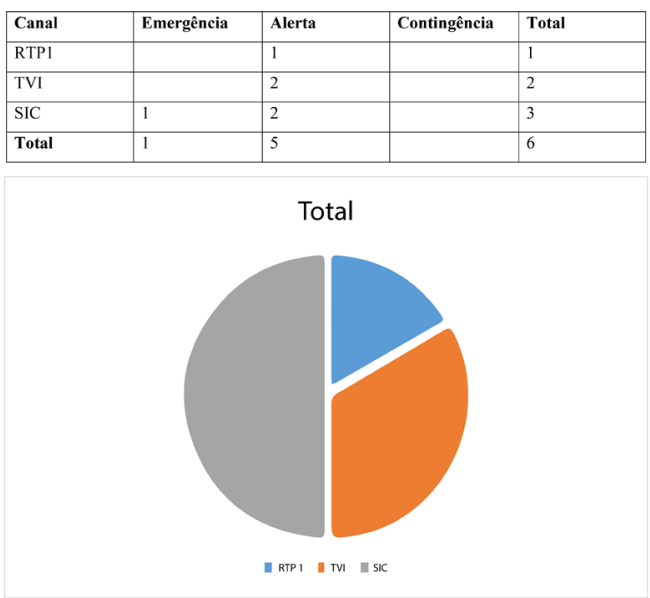

Quadro 3 Quantidade de inserções observadas na intersecção direta entre Cultura/Espetáculo e Covid-19/Saúde. Fonte: e-Telenews - Elaboração própria.

\section{Conclusões}

Os dados permitem concluir que a tematização da Cultura/Espetáculos realizada pelos 3 jornais televisivos foi significativa e diferenciada. As diferenças são relevantes entre os canais, traduzindo diferentes posicionamentos editoriais relativamente à valorização e ao tratamento da cultura. Mas também existe uma variação temática dentro da cultura ao longo do período analisado, e essa é uma tendência transversal. 
A primeira conclusão é a de que a cultura não esteve ausente dos jornais televisivos, mas foi mais tratada pela televisão pública, totalizando um conjunto de inserções (65) muito superior ao da TVI (23) e ao da SIC (19). O telejornal somou sempre mais do dobro (de inserções) do que os jornais televisivos das concorrentes. Entre estes, as diferenças existem, mas são menos relevantes nas duas dimensões (número de peças).

Apesar do menor investimento temático, o Jornal da Noite da SIC conseguiu maiores shares em todos os períodos, confirmando a sua liderança nas audiências neste segmento de programas informativos. A SIC tratou as notícias sobre cultura de forma mais diversa, integrando diferentes assuntos e manteve a sua share para os temas culturais estável ao longo dos 3 períodos, ao contrário da RTP1 que terminou o período de análise com menor share $(21,6 \%)$ do que no início da pandemia $(24,8 \%)$. No caso da RTP1 é curioso assinalar que o período correspondente ao início do estado de contingência (15 de setembro) foi também o que registou uma quebra abrupta no share das notícias sobre cultura apresentadas pelo Telejornal (12,3\%), tendo os níveis voltado a subir nos dias seguintes (16 e 17 de setembro), respetivamente para $23,8 \%$ e $21,5 \%$.

A TVI foi o segundo canal a demonstrar maior interesse pela cultura, mas apresenta o jornal televisivo com menos share. A TVI foi também o canal a dedicar mais proporção de notícias ao assunto 'Situação dos Artistas'. Nesta televisão, a flutuação da cobertura dos temas culturais ao longo do período analisa- 
do foi ascendente: em setembro existiu uma subida considerável $(22,4 \%$ no dia $17 / 09)$ relativamente a março $(16,8 \%$ no dia 17/03). Seria interessante indagar as razões para estas alterações, certamente relacionadas com dimensões de análise das quais não nos ocupamos neste artigo, designadamente a locação de recursos, envolvimento gerado pelas histórias, estratégias de alinhamento e alterações nas rotinas organizacionais.

A segunda conclusão é a de que os três jornais televisivos são atravessados pela mesma dinâmica em relação aos temas culturais, o que indica que as lógicas jornalísticas televisivas são semelhantes e tendem a aproximar os critérios de seleção noticiosa. Se no período de emergência (março) o tema de saúde associado à cultura e espetáculo foi pouco noticiado, já no período de alerta (julho) houve o maior volume de associações do tema Covid-19/Saúde com o da Cultura/Espetáculo. Entre os assuntos mais tratados, destacam-se o papel dos meios de comunicação (TV/Rádios) no cenário cultural, a situação dos artistas que viram as suas oportunidades de atuação profissional presencial reduzirem, as ações de caridade promovidas por (e para) artistas e celebridades, bem como as decisões sobre proibição e limitação de público em festivais e concertos.

A terceira conclusão diz respeito ao detalhe dos assuntos dentro do tema cultura. Constatamos que ultrapassado o trauma inicial gerado pelo lockdown experimentado no estado de emergência e que surtiu efeitos também durante o estado de alerta, as lógicas habituais de tratamento de temas culturais se reinstalaram 
no período de contingência. Observamos uma constância relativamente ao agendamento nos 3 jornais televisivos. Os assuntos relacionados com artistas e personalidades, bem como acontecimentos (especialmente cinemas, teatros e espetáculos) lideram as escolhas. Este alinhamento confirma estudos anteriores (Baptista, 2017) que apontam para um tratamento jornalístico da cultura marcado pela personalização (a glorificação do ideal romântico do criador/autor) e pela sujeição à agenda de eventos (a função de divulgação cultural é dominante). A RTP1 foi a televisão onde a lógica de seleção noticiosa centrada em personalidades foi mais expressiva.

O assunto designado por 'Situação dos Artistas', relacionado com a crise financeira que afetou muitos destes profissionais devido ao encerramento das atividades culturais, teve uma relevância baixa no canal público (9,5\%), esteve ausente do Jornal da Noite (SIC) e recebeu uma maior atenção proporcional no Jornal das 8 (TVI), onde foi mesmo o tema mais tratado. Tendo a nossa análise sido restrita a 9 dias, é possível que os resultados possam variar noutro recorte temporal, mais coincidente com iniciativas específicas relacionadas com a luta política e cívica dos artistas por mais apoios governamentais.

Os dados revelam como a agenda temática cultural dos jornais televisivos é ainda relativamente pobre face à diversidade de dimensões tratáveis e, sobretudo, marcada pelos acontecimentos da atualidade e sem grande capacidade de projeção. Os jornais televisivos não mostram apetência para politizar a cultura 
nem para debater o futuro das atividades culturais no cenário pós-pandemia, desligando-se rapidamente de continuarem a acompanhar as questões relacionadas com a sobrevivência dos artistas e as condições de retorno das atividades culturais. Surpreende a estabilidade da grelha de valores notícia mobilizada para os assuntos culturais e o rápido desaparecimento do enquadramento da pandemia. Para os jornais televisivos, aparentemente, a cultura já seguiu dentro de momentos.

\section{Bibliografia}

e-Telenews (s.d), Mediamonitor, disponível em: http://www.mediamonitor.pt/servicos/telenews.

Baptista, C. (2017). (Org). A Cultura na Primeira Página. Uma Década de Jornalismo Cultural em Portugal. Lisboa: Escritório Editora.

Forde, E. (2003). Journalists with a difference: Producing music journalism. In Cottle, S (ed.) Media Organisations and Production. London: SAGE, pp. 113-130.

Hanitzsch, T., Hanusch, F, Ramaprasad, R. \& de Beer. A. (2019) (Eds). Words of Journalism. Journalistic cultures around the globe. Columbia University Press.

Hanusch, F. (2012) Broadening the focus: The case for lifestyle journalism as a field of scholarly inquiry. Journalism Practice 6(1), pp. 2-11.

Hanusch, F, Hanitzsch, T, Lauerer, C (2017) 'How much love are you going to give this brand?' Lifestyle journalists on commercial influences in their work. Journalism 18(2), pp. 141-158.

Harries, G., Wahl-Jorgensen, K. (2007) The culture of arts journalists. Journalism 8(6), pp. 619-639. 
Hartley, J. (2009). Popular Reality: Journalism and Popular Culture. Bloomsbury Academic.

Hartley, J. (2019). What is Television. In Shimpach, S. (Ed). The Routledge Companion to Global Television. New York: Routledge.

Hellman, H. \& Jaakkola, M. (2012) From aesthetes to reporters: The paradigm shift in Finland. Journalism 13(6), pp. 783-801.

Hovden, J. \& Kristensen. N. (2018). The cultural journalist around the globe: A comparative study of characteristics, role perceptions, and perceived influences. Journalism. August 2028.

Jaakkola, M. (2015). The contested autonomy of arts and journalism: Change and continuity of the dual professionalism of cultural journalism. PhD Thesis. Tampere: University of Tampere.

Kersten, A. \& Janssen, S. (2016). Trends in Cultural Journalism. The development of film coverage in cross-national perspective, 1955-2005. Journalism Practice 11 (7), pp. 840-856.

Kristensen, N. \& Riegert, K. (2017) (Eds). Cultural Journalism in the Nordic Countries. Nordicom.

Ong, J. \& Negra, D. (2020). The Media (Studies) of the Pandemic Moment: Introduction to the 20th Anniversary Issue. Television \& New Media, September 2020, pp. 555-561.

Sarrimo, C. (2017). The press crisis and its impact on Swedish arts journalism: Autonomy loss, a shifting paradigm and a 'journalistification' of the profession. Journalism 18, pp. 664-679.

Stuart, H. (1985). Signification, representation, ideology: Althusser and the post-structuralist debates. Critical Studies in Mass Communication, 2:2, pp. 91-114. 